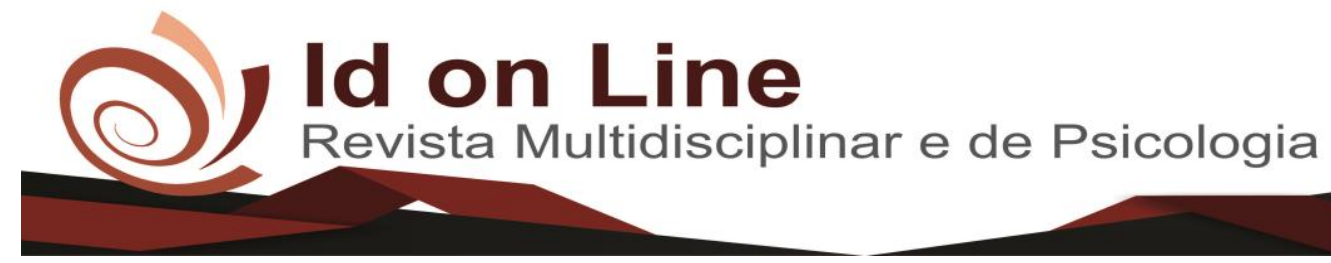

DOI: 10.14295/idonline.v14i53.2849

Artigo

\title{
Depressão em Idosos: \\ Implicações sociais e outras intercorrências
}

\author{
Gustavo Leitao de Figueiredo Medeiros ${ }^{1}$; Miguel Aguila Toledo \\ Milena Nunes Alves de Sousa ${ }^{3}$
}

\begin{abstract}
Resumo: A depressão pode atingir todas as faixas etárias, principalmente o idoso e decorre de diversos fatores que envolvem o processo de envelhecimento. Diante disso, objetiva-se identificar, na literatura cientifica, as implicações sociais e outras intercorrências da depressão em idosos, sob o prisma da atenção primária à saúde. Este estudo desenvolveu-se por meio de uma revisão integrativa. Quanto aos critérios de inclusão, considerou-se estudos publicados em português e espanhol e entre os anos de 2018 a 2020. Dos estudos incluídos, foram extraídas informações de forma categorizada quanto ao ano, título, base de dados, periódico, idioma, país, método e principais achados. Ao fim, reuniu-se 18 estudos que atenderam os critérios de elegibilidade definidos, e destes houve prevalência para os anos de 2018 e 2019 de publicação; não houve predominância quanto ao veículo de publicação; e houve prevalência de estudos transversais e no idioma português. Ao fim, constatou-se os fatores significativamente associados à depressão em idosos e as implicações sociais e outras intercorrências relevantes ao estudo da depressão, e diante dessa problemática, considera-se necessário uma maior prudência, no atendimento no âmbito da atenção primária.
\end{abstract}

Palavras - Chave: Transtornos de Adaptação; Atenção Primária à Saúde; Atenção Integral à Saúde; Relações Interpessoais.

\section{Depression in the Elderly: Social implications and other complications}

\begin{abstract}
Depression can affect all age groups, especially the elderly, and results from several factors that involve the aging process. Therefore, the objective is to identify, in the scientific literature, social information and other complications of depression in the elderly, from the perspective of primary health care. This study was discovered through an integrative review. As for the included criteria, studies published in Portuguese and Spanish and between the years 2018 to 2020 were considered. From the included studies, information was extracted in a categorized way regarding the year, title, database, journal, language, country, method and main findings. At the end, 18 studies met the defined eligibility criteria, and these prevailed for the years 2018 and 2019 of publication; there was no predominance regarding the publication vehicle; and prevalence of cross-sectional studies and in Portuguese. In the end, it was found the secondary factors associated with depression and as social derivatives and other complications relevant to the study of depression, and in view of this problem, it is considered necessary to be more prudent in the care provided in primary care.
\end{abstract}

Keywords: Adaptation Disorders; Primary Health Care; Comprehensive Health Care; Interpersonal Relationships.

\footnotetext{
${ }^{1}$ Médico formado pela FCM PB, Pós graduação em Psiquiatria pela universidade Estácio de Sá, Médico Residente em Medicina de Família e Comunidade, Especialização em Preceptoria de Residência Médica pelo Hospital Sírio Libanês. E-mail: gustavolfm1@ hotmail.com

${ }^{2}$ Mestre em infectologia. E-mail: migueltoledo@ fiponline.edu.br

${ }^{3}$ Doutorado e Pós-Doutorado em Promoção de Saúde. E-mail: milenanunes@ fiponline.edu.br
}

474 Id on Line Rev. Mult. Psic. V.14 N. 53, p. 474-483, Dezembro/2020 - ISSN 1981-1179 Edição eletrônica em http://idonline.emnuvens.com.br/id 


\section{Introdução}

A depressão é uma síndrome decorrente de um conjunto de mecanismos patogênicos e etiológicos, oriundos de um déficit de neurotransmissores monoaminérgicos, responsáveis, por exemplo, pelo aumento da serotonina, dopamina, adrenalina, e outros, na fenda sináptica, resultando em sensações de conforto, humor, apetite, prazer e bem estar (físico e emocional) (AGUIAR; CASTRO; CARVALHO, 2011; RAZZOUK, 2016; SOUSA et al., 2017).

Esta síndrome pode atingir todas as faixas etárias, principalmente o idoso. As mudanças ocorridas com o envelhecimento proporcionam inúmeras limitações e perdas, a exemplo, a aposentadoria de trabalho, a morte de entes queridos, os problemas médicos, tendo como consequências o isolamento e a auto depreciação (GUIMARÃES et al., 2019).

A depressão é a quarta causa mais importante de inaptidão mundial, e espera-se que se torne a segunda causa mais importante até 2020, sendo por isso considerada um grave e importante problema de saúde, sendo por isso, além do tratamento, a urgência em se debater sobre assunto. (ABELHA, 2014; RAZZOUK, 2016).

Tendo em vista outras implicações que podem culminar a depressão em idosos, assim como as complicações desta síndrome neste grupo, este estudo tem o objetivo de identificar, na literatura científica, as implicações sociais e outras intercorrências relevantes ao estudo da depressão em idosos, sob o prisma da atenção primária à saúde.

Ressalta- se a importância de se realizar pesquisas relacionadas à depressão em idosos, em razão de promover discussões e reflexões principalmente sobre aspectos preventivos da depressão em idosos. No mais, acredita-se que este estudo contribuirá como fonte de consulta e atualização para os profissionais da área da psiquiatria e de áreas afins.

\section{Métodos}

Este estudo desenvolveu-se por meio de uma revisão integrativa, a qual permite, por meio de distintas metodologias, de acordo com que assinalam Souza; Silva; Carvalho (2010), uma atualização sobre a temática.

As etapas metodológicas desta pesquisa abrangeram a elaboração da pergunta norteadora, a definição dos bancos de dados que serviram como instrumento de pesquisa, assim como a definição dos descritores, a definição dos critérios de elegibilidade, e a análise e inclusão dos estudos (SOUZA; SILVA; CARVALHO; 2010, SOUSA, 2016). 
A pergunta norteadora do estudo é a seguinte: "Quais as implicações sociais e outras consequências relacionadas à depressão no idoso?". Quanto aos procedimentos de coleta de dados, este foi realizado no período de maio a junho do ano de 2020 nas bases de dados, US National Library of Medicine National Institutes of Health (PUBMED), Medical Literature Analysis and Retrieval System Online (MEDLINE) e Literatura Latino-Americana e do Caribe em Ciências da Saúde (LILACS) e Scientific Electronic Library Online (SCIELO), abrangendo estudos publicados em português e espanhol. Utilizaram-se os seguintes descritores, em português e espanhol, respectivamente: Transtornos de adaptação; Atenção primária à saúde; Atenção integral à saúde; Relações interpessoais. Trastornos de adaptación; Primeros auxilios; Atención integral de salud; Relaciones interpersonales.

Quanto aos critérios de inclusão, consideraram-se estudos publicados em português e espanhol e entre os anos de 2018 a 2020. Foram excluídos artigos incompletos, como também teses, editoriais, estudos que não se relacionem as palavras chaves, artigos repetidos nas diferentes bases de dado, além daqueles que não apresentarem relevância para o presente estudo.

Figura 01: Fluxograma de seleção dos artigos.

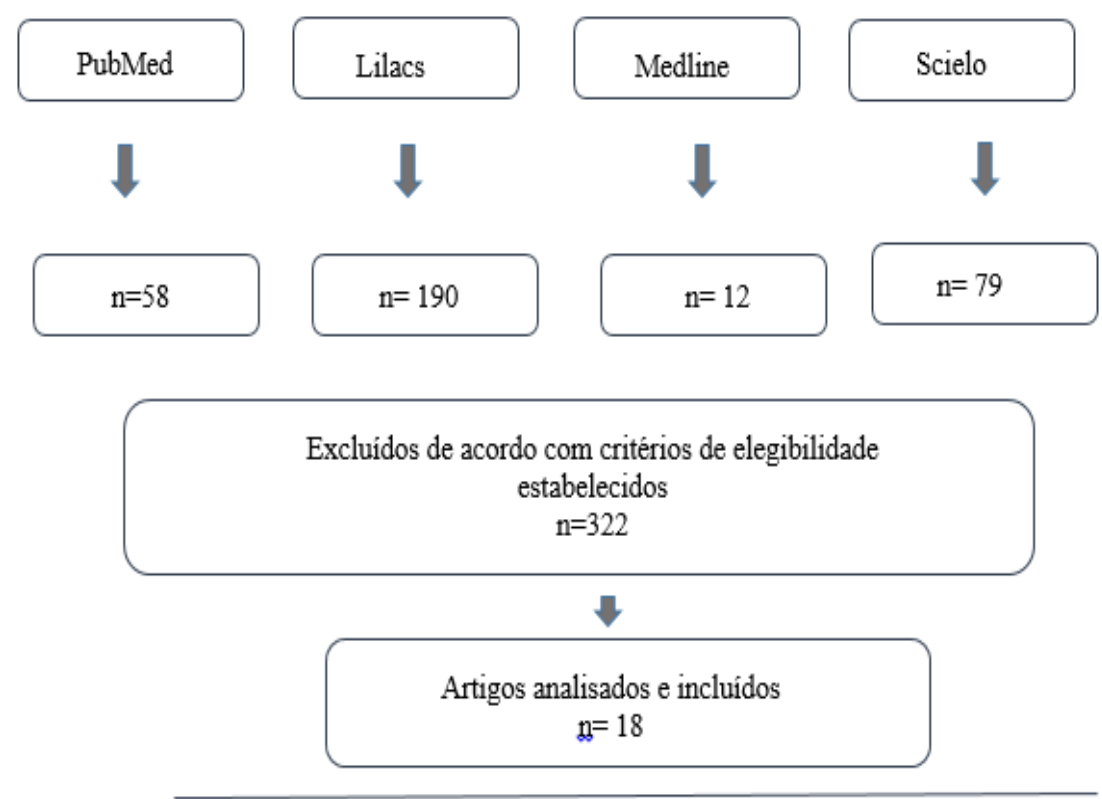

Fonte: Elaborado pelos autores (2020).

Conseguiu-se reunir 339 estudos relevantes nos bancos de dados. Destes 106 foram excluídos com base nos títulos e resumos; 208 foram excluídos com base no ano de publicação; e 7 foram excluídos por serem dissertações e estudos com informações incompletas. 
Ao fim, reuniram-se 18 estudos que atenderam os critérios de elegibilidade definidos. Dos estudos reunidos, sistematizaram-se informações sobre quanto ao ano de publicação, o título do estudo, a base de dados e periódicos indexados, o idioma e o país, o método, e os principais achados.

Dos estudos incluídos, foram extraídas informações de forma categorizadas quanto ao ano, título, base de dados, periódico, idioma, país, método e principais achados.

\section{Resultados}

Constata-se que os anos de prevalência das publicações foram os de 2018 (33\%; n=6) e 2019 (61\%; n=11). No que tange aos periódicos que serviram de veículos para a publicação dos artigos não houve predominância, sendo 18 diferentes periódicos. Com relação ao enquadramento dos estudos maioria eram estudos transversais $(59 \% ; n=10)$ (Tabela 1).

Quanto ao ano, 61\% ( $\mathrm{n}=11)$ foram publicados em 2019, em 18 periódicos distintos, os estudos transversais foram os tipos mais comuns $(59 \% ; n=10)$. Sobre o idioma, o português foi prevalente $(61 \% ; n=11)$ e a base de dados mais prevalente foi a Literatura Latino-Americana e do Caribe em Ciências da Saúde (LILACS) $(61 \%$; n=11) (Tabela 1).

Tabela 1 - Caracterização dos estudos incluídos de acordo com autor e ano, título, base de dados, periódico, idioma, país e método.

\begin{tabular}{|c|c|c|c|c|c|c|}
\hline Autor/ ano & Título & $\begin{array}{c}\text { Base de } \\
\text { dados }\end{array}$ & Periódico & Idioma & País & Método \\
\hline $\begin{array}{c}\text { Aravena, } \\
\text { Gajardo e } \\
\text { Saguez } \\
(2019)\end{array}$ & $\begin{array}{l}\text { Salud mental de } \\
\text { hombres mayores } \\
\text { en Chile: una } \\
\text { realidad por } \\
\text { priorizar }\end{array}$ & SCIELO & $\begin{array}{c}\text { Revista } \\
\text { Panamericana } \\
\text { de La Salude } \\
\text { Publica }\end{array}$ & Espanhol & Chile & $\begin{array}{l}\text { Revisão } \\
\text { narrativa } \\
\text { retrospecti } \\
\text { va }\end{array}$ \\
\hline $\begin{array}{c}\text { Calderón } \\
\text { (2018) }\end{array}$ & $\begin{array}{c}\text { Epidemiología de la } \\
\text { depresión en el } \\
\text { adulto mayor }\end{array}$ & SCIELO & $\begin{array}{c}\text { Revista } \\
\text { Médica } \\
\text { Herediana }\end{array}$ & Espanhol & Equador & $\begin{array}{l}\text { Revisão } \\
\text { narrativa }\end{array}$ \\
\hline $\begin{array}{l}\text { Ferraiuoli e } \\
\text { Ferreira } \\
(\mathbf{2 0 1 8})\end{array}$ & $\begin{array}{l}\text { O outro lado da } \\
\text { "melhor idade": } \\
\text { Depressão e } \\
\text { Suicídio em Idosos }\end{array}$ & SCIELO & $\begin{array}{l}\text { Revista } \\
\text { Perspectivas } \\
\text { Humanas \& } \\
\text { Sociais } \\
\text { Aplicadas }\end{array}$ & Português & Brasil & $\begin{array}{l}\text { Revisão } \\
\text { narrativa }\end{array}$ \\
\hline $\begin{array}{c}\text { Frutuoso et } \\
\text { al. (2019) }\end{array}$ & $\begin{array}{c}\text { Idosos } \\
\text { institucionalizados } \\
\text { e depressão: } \\
\text { rastreamento dos } \\
\text { sintomas }\end{array}$ & SCIELO & $\begin{array}{c}\text { Revista } \\
\text { Enfermagem } \\
\text { Brasil }\end{array}$ & Português & Brasil & $\begin{array}{c}\text { Estudo } \\
\text { descritivo }\end{array}$ \\
\hline $\begin{array}{l}\text { Guimarães } \\
\text { et al. (2019) }\end{array}$ & $\begin{array}{c}\text { Sintomas } \\
\text { depressivos e } \\
\text { fatores associados }\end{array}$ & SCIELO & $\begin{array}{l}\text { Ciência \& } \\
\text { Saúde } \\
\text { Coletiva }\end{array}$ & Português & Brasil & $\begin{array}{l}\text { Estudo } \\
\text { transversal }\end{array}$ \\
\hline
\end{tabular}




\begin{tabular}{|c|c|c|c|c|c|c|}
\hline & $\begin{array}{c}\text { em idosos } \\
\text { residentes em } \\
\text { instituição de longa } \\
\text { permanência }\end{array}$ & & & & & \\
\hline $\begin{array}{l}\text { Molina et al. } \\
\qquad(2020)\end{array}$ & $\begin{array}{l}\text { Factores de riesgo } \\
\text { asociados a la } \\
\text { conducta suicida en } \\
\text { el adulto mayo }\end{array}$ & LILACS & Medicentro & Espanhol & Cuba & $\begin{array}{l}\text { Estudo } \\
\text { transversal }\end{array}$ \\
\hline $\begin{array}{l}\text { Nascimento } \\
\text { e Batistoni } \\
(2019)\end{array}$ & $\begin{array}{c}\text { Depressão e } \\
\text { fragilidade na } \\
\text { velhice: uma } \\
\text { revisão narrativa } \\
\text { das publicações de } \\
\text { 2008-2018 }\end{array}$ & LILACS & $\begin{array}{l}\text { Interface } \\
\text { (Botucatu) }\end{array}$ & Português & Brasil & $\begin{array}{l}\text { Revisão } \\
\text { Narrativa }\end{array}$ \\
\hline Park (2019) & $\begin{array}{c}\text { Tipo mais } \\
\text { prevalente de abuso } \\
\text { aos idosos e sua } \\
\text { correlação com } \\
\text { depressão do idoso }\end{array}$ & LILACS & $\begin{array}{l}\text { Acta Paulista } \\
\text { de } \\
\text { Enfermagem }\end{array}$ & Português & $\begin{array}{l}\text { Coréia } \\
\text { do Sul }\end{array}$ & $\begin{array}{l}\text { Estudo } \\
\text { transversal }\end{array}$ \\
\hline $\begin{array}{l}\text { Puello et al. } \\
\text { (2019) }\end{array}$ & $\begin{array}{l}\text { Sentimientos de } \\
\text { depresión en } \\
\text { personas mayores } \\
\text { beneficiarios de un } \\
\text { programa de } \\
\text { extensión solidaria } \\
\text { "salud familiar" } \\
\text { Córdoba Colombia }\end{array}$ & LILACS & $\begin{array}{c}\text { Revista } \\
\text { Avances en } \\
\text { Salud }\end{array}$ & Espanhol & $\begin{array}{c}\text { Colômbi } \\
\text { a }\end{array}$ & $\begin{array}{c}\text { Estudo } \\
\text { transversal }\end{array}$ \\
\hline $\begin{array}{l}\text { Quintana et } \\
\text { al. (2019) }\end{array}$ & $\begin{array}{l}\text { Caracterización de } \\
\text { las condiciones de } \\
\text { salud de los adultos } \\
\text { mayores en Centros } \\
\text { Geriátricos de la } \\
\text { ciudad de Loja. } \\
\text { Ecuador, } 2017\end{array}$ & LILACS & $\begin{array}{c}\text { Revista } \\
\text { Habanera de } \\
\text { Ciencias } \\
\text { Médicas }\end{array}$ & Espanhol & Equador & $\begin{array}{l}\text { Estudo } \\
\text { transversal }\end{array}$ \\
\hline $\begin{array}{l}\text { Ramos et al. } \\
\qquad(2019)\end{array}$ & $\begin{array}{c}\text { Fatores associado à } \\
\text { depressão em } \\
\text { idosos }\end{array}$ & PUBMED & $\begin{array}{c}\text { Revista } \\
\text { Eletrônica } \\
\text { Acervo Saúde }\end{array}$ & Português & Brasil & $\begin{array}{l}\text { Revisão } \\
\text { Integrativa }\end{array}$ \\
\hline $\begin{array}{c}\text { Rodríguez et } \\
\text { al. (2018) }\end{array}$ & $\begin{array}{c}\text { Funcionalidad } \\
\text { familiar y depresión } \\
\text { en adultos mayores } \\
\text { en una institución } \\
\text { de salud de Ciudad } \\
\text { Victoria }\end{array}$ & LILACS & $\begin{array}{l}\text { Revista de } \\
\text { Enfermería } \\
\text { Neutrológica }\end{array}$ & Espanhol & México & $\begin{array}{c}\text { Estudo } \\
\text { transversal }\end{array}$ \\
\hline $\begin{array}{l}\text { Rossetti et } \\
\text { al. (2018) }\end{array}$ & $\begin{array}{c}\text { Fragilidade, } \\
\text { sintomas } \\
\text { depressivos e } \\
\text { sobrecarga de } \\
\text { idosos cuidadores } \\
\text { em contexto de alta } \\
\text { vulnerabilidade } \\
\text { social }\end{array}$ & LILACS & $\begin{array}{c}\text { Texto \& } \\
\text { Contexto } \\
\text { Enfermagem }\end{array}$ & Português & Brasil & $\begin{array}{l}\text { Estudo } \\
\text { transversal }\end{array}$ \\
\hline $\begin{array}{c}\text { Saintrain et } \\
\text { al. (2018) }\end{array}$ & $\begin{array}{c}\text { Idosos com } \\
\text { depressão: uma } \\
\text { análise dos fatores } \\
\text { de }\end{array}$ & LILACS & $\begin{array}{l}\text { Revista } \\
\text { Brasileira de } \\
\text { Promoção à } \\
\text { Saúde }\end{array}$ & Português & Brasil & $\begin{array}{l}\text { Estudo } \\
\text { transversal }\end{array}$ \\
\hline $\begin{array}{l}\text { Santos e } \\
\text { Santos } \\
(2019)\end{array}$ & $\begin{array}{l}\text { institucionalização } \\
\text { e apoio familiar } \\
\text { Depressão em } \\
\text { Idosos }\end{array}$ & SCIELO & $\begin{array}{l}\text { Revista de } \\
\text { Saúde Da } \\
\text { ReAGES }\end{array}$ & Português & Brasil & $\begin{array}{l}\text { Revisão } \\
\text { Sistemátic } \\
\text { a }\end{array}$ \\
\hline
\end{tabular}




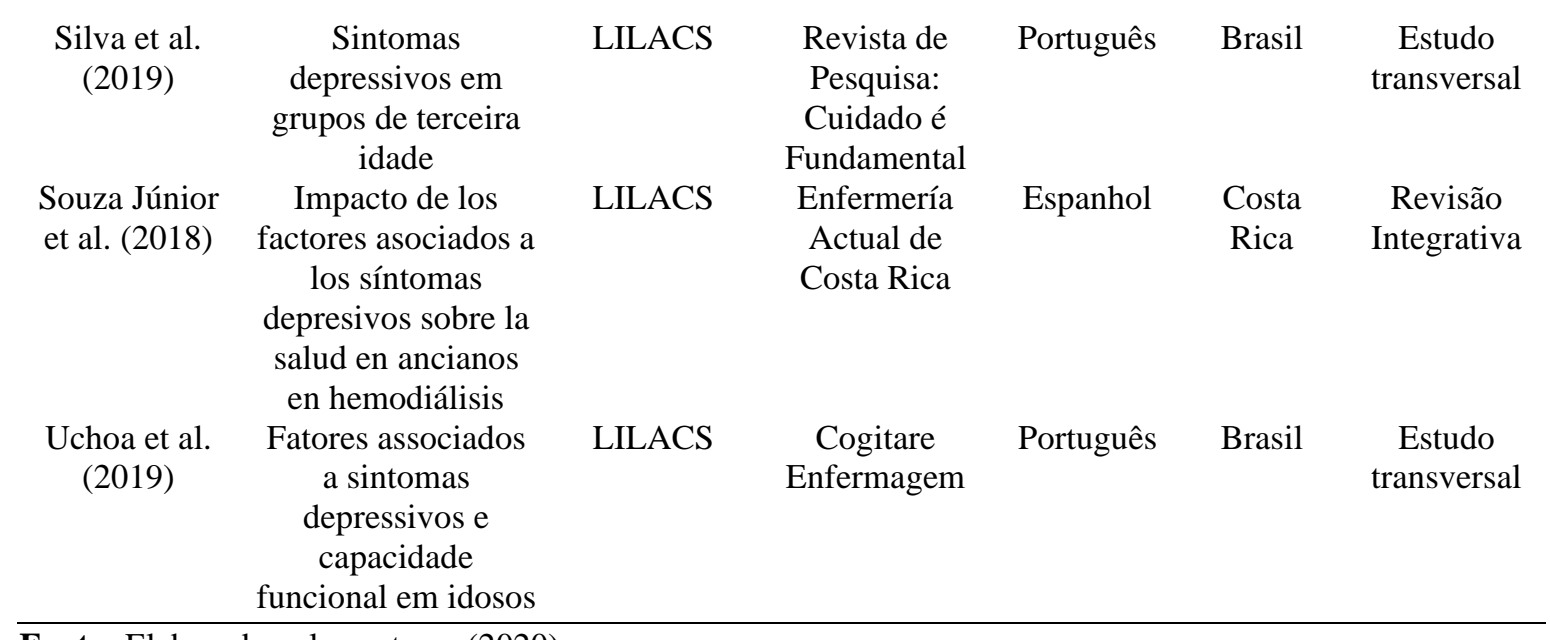

Fonte: Elaborado pelos autores (2020).

$\mathrm{Na}$ tabela 2, expõe-se a categorização quanto às implicações sociais e outras intercorrências acerca da depressão em idosos, com base nos estudos aqui inclusos. A maioria dos estudos enquadrou-se na categoria $1(83,3 \% ; n=15)$.

Tabela 2 - Categorização dos estudos quanto às implicações sociais e outras intercorrências da depressão em idosos.

\begin{tabular}{ccc}
\hline Categoria & $\mathbf{N}(\%)$ & Autores \\
\hline Implicações sociais da & $15(83,3 \%)$ & Aravena, Gajardo e Saguez \\
depressão em idosos & Calderón (2018) \\
(solidão; ausência de & Ferraiuoli e Ferreira (2018) \\
convívio familiar; e & Frutuoso et al. (2019) \\
isolamento) & Guimarães et al. (2019) \\
& Nascimento e Bastitoni \\
& $(2019)$ \\
& Puello et al. (2019) \\
& Quintana et al. (2019) \\
& Ramos et al. (2019) \\
& Rodriguez et al. (2018) \\
& Rossetti et al. (2018) \\
& Saintrain et al. (2018) \\
& Santos e Santos (2019) \\
& Silva et al. (2019) \\
& Souza Júnior et al. (2018) \\
\hline Outras intercorrências (maus & Aravena, Gajardo e Saguez \\
tratos; dependência / & $(2019)$ \\
ausência de autonomia; & Guimarães et al. (2019) \\
fragilidade física; & Molina et al. (2020) \\
dificuldades para & Park (2019) \\
desempenho profissional; & Quintana et al. (2019) \\
perdas financeiras; & Souza Júnior et al. (2018) \\
demência). & Uchoa et al. (2019) \\
\hline
\end{tabular}

Fonte: Elaborado pelos autores (2020). 


\section{Discussão}

Para mapear os principais achados dos estudos incluídos nesta revisão integrativa, avançou-se para discussão dos achados. A maioria dos estudos contemplou as implicações sociais (CALDERÓN, 2018; FERRAIUOLI; FERREIRA, 2018; RODRIGUEZ et al., 2018; ROSSETTI et al., 2018; SAINTRAIN et al., 2018; SOUZA JÚNIOR et al., 2018; ARAVENA; GAJARDO; SAGUEZ, 2019; FRUTUOSO et al., 2019; GUIMARÃES et al. 2019; NASCIMENTO; BASTITONI, 2019; PUELLO et al., 2019; QUINTANA et al., 2019; RAMOS et al., 2019; SANTOS; SANTOS, 2019; SILVA et al., 2019).

No mais, outras pesquisas contemplaram outras intercorrências acerca da depressão em idosos (SOUZA JÚNIOR et al., 2018; ARAVENA; GAJARDO; SAGUEZ, 2019; GUIMARÃES et al. 2019; PARK, 2019; QUINTANA et al., 2019; UCHOA et al., 2019; MOLINA et al., 2020).

De acordo com Saintrain et al. (2018), o tempo de institucionalização, a carência das relações interpessoais, a solidão e o fato de o idoso de não receber visitas de familiares neste período constituem fatores de risco para a depressão. Souza Júnior et al. (2018) esclarecem que os impactos dos fatores associados à depressão na saúde de idosos perpassam pelos aspectos físicos, psicológicos, emocionais, comportamentais e sociais. Park (2019) defende que o abuso emocional e o abuso verbal são significativamente relacionados à depressão do idoso.

Para Rodriguez et al. (2018) há relação entre a funcionalidade familiar e a depressão em idosos. Já Rossetti et al. (2018) afirmam haver correlação entre fragilidade e sintomas depressivos. Diante disso, faz-se necessária a abordagem dos profissionais de saúde no sentido de identificar precocemente a fragilidade e os sintomas depressivos. Santos e Santos (2019) complementam aos dois últimos estudos citados, ao apontarem também que os fatores que se correlacionam com a depressão em idosos variam conforme diferentes características populacionais.

Quintana et al. (2019) e Nascimento e Bastitoni (2019) revelam que a prevalência de depressão em idosos são do sexo feminino, com ensino fundamental ou médio e idade média acima de 80 anos. Prevaleceram indivíduos que não apresentam comorbidade, déficits visuais e auditivos, fragilidade física, dependência funcional para realizar as atividades básicas e instrumentais da vida cotidiana, comprometimento cognitivo e depressão.

Guimarães et al. (2019) acrescentam que há alta prevalência de sintomas depressivos em idosos institucionalizado. Diante disso, Frutuoso et al. (2019) defendem que seja primordial 
a criação de programas para idosos institucionalizados, e que tais programas visem a promoção social, cultural, de lazer, de esporte e educacional.

No mais, Calderón (2018) e Ferraiuoli e Ferreira (2018) expõem que a depressão em idosos tem características próprias e o impacto na qualidade de vida, e se relacionam com comorbidades, além de haver uma ideação suicida evidente. Para os autores, a depressão tornase muito difícil de ser diagnosticada por ter seus sintomas facilmente confundidos com as queixas somáticas de um processo de envelhecimento normal. Devido a exposto, recomendase a detecção precoce na atenção primária.

Acerca da ideação suicida, Molina et al. (2020) complementam que os fatores de risco para depressão em idosos como fragilidade física, dependência funcional, instrumental da vida cotidiana e financeira, levam facilmente a uma conduta suicida.

Assim, se faz necessário agir de modo preventivo, junto aos idosos (RAMOS et al., 2019). A prática e o desenvolvimento de atividades de lazer têm-se mostrado na vida dos idosos como um fator de grande importância, visto que, de acordo com Uchoa et al. (2019) a não realização de atividades instrumentais de vida diária está fortemente associado à presença de sintomas depressivos e idade avançada.

Com base na discussão realizada, nota-se que a depressão é condição clínica frequente no idoso, portanto, um distúrbio que merece atenção especial, devido as suas implicações neste grupo etário que, por uma série de razões, necessita de cuidados especiais. Ressalta-se que a depressão, por ser agravo de saúde mental, pode reduzir imensamente a qualidade de vida e antecipar sua morte.

\section{CONCLUSÃO}

Esta revisão integrativa possibilitou caracterizar a produção científica acerca dos fatores significativamente associados à depressão em idosos e as implicações sociais e outras intercorrências relevantes ao estudo da depressão. Sendo assim, percebe-se que a depressão em idosos implica vários fatores, tais como carência das relações interpessoais, ausência de presença familiar para aqueles idosos institucionalizados, dependência funcional, negligência, e abusos físicos e psicológicos.

Diante desse quadro, se faz necessário uma maior prudência, no atendimento no âmbito da atenção primária à saúde a este grupo populacional, visto que um quadro depressivo resulta em vulnerabilidade biopsicossocial, podendo levar inclusive ao suicídio. 
Sendo assim, condensar evidências científicas sobre a depressão em idosos é de grande valia, e poderá servir de instrumento de reflexão e debate sobre estratégias preventivas que aprimorem a prática daqueles envolvidos no cuidado com o idoso a fim de promover o bem estar destes.

\section{Referências}

ARAVENA, J.M; GAJARDO, J; SAGUEZ, R. Salud mental de hombres mayores en Chile: una realidad por priorizar. Revista Panamericana de Salud Pública, v.42, n7, 2019.

AGUIAR, C.C.; CASTRO, T.R.; CARVAlHO, A.F. Drogas Antidepressivas. Acta Medica Portuguesa, v.24, n.1, p. 91-98, 2011.

ABELHA, L. Depressão, uma questão de saúde pública. Cadernos de Saúde Coletiva, Rio de Janeiro, v.22, n.3, p. 223, 2014.

CALDERÓN, M. Epidemiología de la depresión en el adulto mayor. Revista Medica Herediana, v. 29, p. 182-191, 2018.

FERRAIUOLI, C.; FERREIRA, S.M.R.R. O outro lado da "melhor idade": depressão e suicídio em idosos. Perspectiva Online: Humanas \& Sociais Aplicadas, v.18, n7, p.43-53, 2017.

FRUTUOSO, E.A. et al. Idosos institucionalizados e depressão: rastreamento dos sintomas. Enfermagem Brasil, v.18, n.3, 2019.

GUIMARÃES, L.A. et al. Sintomas depressivos e fatores associados em idosos residentes em instituição de longa permanência. Ciência \& Saúde Coletiva, v.24, n.9, p. 3275-3282, 2019.

MOLINA, L. et al. Factores de riesgo asociados a la conducta suicida en el adulto mayor / Risk factors associated with suicidal behavior in the elderly. MediCentro, v.24, n.1, p. 54-67, 2020.

NASCIMENTO, P.P.P.; BASTITONI, S.S.T. Depressão e fragilidade na velhice: uma revisão narrativa das publicações de 2008-2018. Interface, v.23, 2019.

PARK, E.O. Tipo mais prevalente de abuso aos idosos e sua correlação com depressão do idoso. Acta Paulista de Enfermagem, v.32, n.1, p. 95-100, 2019.

PUELLO, A.E. et al. Sentimientos de depresión en personas mayores beneficiarios de un programa de extensión solidaria "salud familiar" Córdoba Colombia. La Revista Avances en Salud de la Universidad de Córdoba, v.3, n.2, p.27-32, 2019.

QUINTANA, T.R. et al. Caracterización de las condiciones de salud de los adultos mayores en Centros Geriátricos de la ciudad de Loja. Ecuador, 2017. Revista Habanera de Ciencias Médicas, v.18, n.1, p. 138-149, 2019. 
RAZZOUK, D. Por que o Brasil deveria priorizar o tratamento da depressão na alocação dos recursos da Saúde?. Epidemiologia e Serviços de Saúde, v.25, n.4, p. 845-848, 2016.

RAMOS, F.B. et al. Fatores associados à depressão em idoso. Revista Eletrônica Acervo Saúde, v supl., n. 19, p. 1-8, 2019.

RODRÍGUEZ, G. et al. Funcionalidad familiar y depresión en adultos mayores en una institución de salud de Ciudad Victoria. Revista de Enfermaria Neurológica, v.17, n.2, p. 3341, 2018.

ROSSETTI, E.S. et al. Fragilidade, sintomas depressivos e sobrecarga de idosos cuidadores em contexto de alta vulnerabilidade social. Texto \& Contexto - Enfermagem, v.27, n.3, p. 1-11, 2018 .

SANTOS; M.A.M.; SANTOS, M.C. Depressão em Idosos. Revista de Saúde ReAGES, v.2, n.4, 2019.

SAINTRAIN, M.V.L.; et al. Idosos com depressão: uma análise dos fatores de institucionalização e apoio familiar. Revista Brasileira de Promoção em Saúde, v.31, n.4, p.1-7, 2018.

SILVA, A.L.A.G; FERNANDES, F.E.C.V; OLIVEIRA, M.M.A; et al. Sintomas depressivos em grupos de terceira idade. Revista de Pesquisa: Cuidado é Fundamental (Online), v.11, p. 297-303, 2019.

SOUSA, K.A. et al. Prevalência de sintomas de depressão em idosos assistidos pela Estratégia de Saúde da Família. REME - Revista Mineira de Enfermagem, v.21, p.1-7, 2017.

SOUSA, M. N. A. Revisão Integrativa da Literatura: esclarecendo o método. In: SOUSA, M. N. A.; SANTOS, E. V. L. Medicina e pesquisa: um elo possível. Cutitiba: Editora CRV, 2016. p. 345-358,

SOUZA JÚNIOR, E.V. et al. Impacto dos fatores associados à sintomatologia depressiva na saúde de idosos em hemodiálise. Enfermaría Actual em Costa Rica, v.35, p. 159-172, 2018.

SOUZA, M.T.; SILVA, M.D.; CARVALHO, R. Revisão integrativa: o que é e como fazer. Einstein, v. 8, n.1. p. 102-106, 2010.

UCHOA, V.S. et al. Fatores associados a sintomas depressivos e capacidade funcional em idosos. Cogitare Enfermagem, v.24, 2019.

\section{Como citar este artigo (Formato ABNT):}

MEDEIROS, Gustavo Leitao de Figueiredo; TOLEDO, Miguel Aguila; SOUSA, Milena Nunes Alves de. Depressão em Idosos: Implicações sociais e outras intercorrências. Id on Line Rev.Mult. Psic., Dezembro/2020, vol.14, n.53, p. 474-483. ISSN: 1981-1179.

Recebido: 08/12/2020;

Aceito: $12 / 12 / 2020$. 